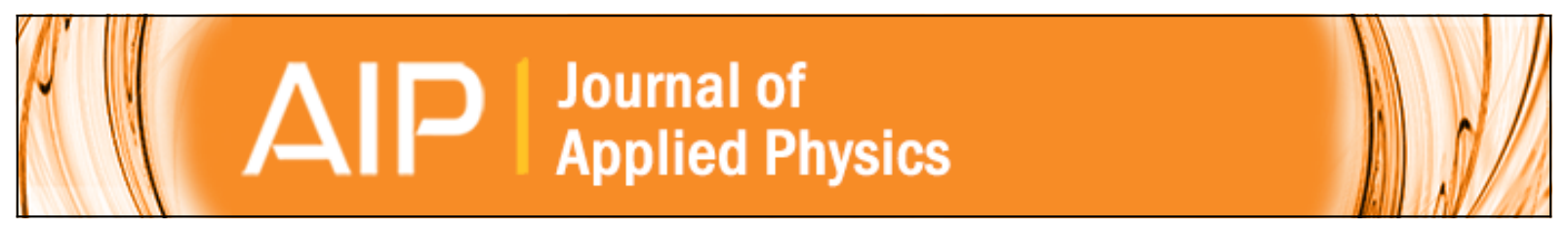

\title{
Numerical modeling of highly doped Si:P emitters based on Fermi-Dirac statistics and self-consistent material parameters
}

Pietro P. Altermatt, Jürgen O. Schumacher, Andres Cuevas, Mark J. Kerr, Stefan W. Glunz, Richard R. King, Gernot Heiser, and Andreas Schenk

Citation: Journal of Applied Physics 92, 3187 (2002); doi: 10.1063/1.1501743

View online: http://dx.doi.org/10.1063/1.1501743

View Table of Contents: http://scitation.aip.org/content/aip/journal/jap/92/6?ver=pdfcov

Published by the AIP Publishing

\section{Articles you may be interested in}

Deposition temperature independent excellent passivation of highly boron doped silicon emitters by thermal atomic layer deposited $\mathrm{Al} 2 \mathrm{O} 3$

J. Appl. Phys. 114, 094505 (2013); 10.1063/1.4819970

Empirical determination of the energy band gap narrowing in highly doped $\mathrm{n}+$ silicon

J. Appl. Phys. 114, 044508 (2013); 10.1063/1.4816694

Nickel solubility in intrinsic and doped silicon

J. Appl. Phys. 97, 023505 (2005); 10.1063/1.1836852

Below bulk-band-gap photoluminescence at room temperature from heavily P-and B-doped Si nanocrystals J. Appl. Phys. 94, 1990 (2003); 10.1063/1.1590409

Surface recombination velocity of phosphorus-diffused silicon solar cell emitters passivated with plasma enhanced chemical vapor deposited silicon nitride and thermal silicon oxide

J. Appl. Phys. 89, 3821 (2001); 10.1063/1.1350633

\section{AIP $\mid$ APL Photonics}

APL Photonics is pleased to announce Benjamin Eggleton as its Editor-in-Chief

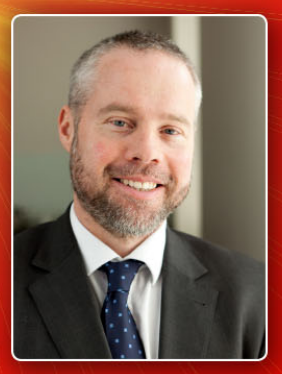




\title{
Numerical modeling of highly doped Si:P emitters based on Fermi-Dirac statistics and self-consistent material parameters
}

\author{
Pietro P. Altermatt \\ Centre for Photovoltaic Engineering, University of New South Wales, Sydney 2052, Australia \\ and Inianga Consulting, 92/125 Oxford Street, Bondi Junction NSW 2022, Australia
}

Jürgen O. Schumacher

Fraunhofer Institute for Solar Energy Systems (ISE), Heidenhofstrasse 2, 79110 Freiburg, Germany

Andres Cuevas and Mark J. Kerr

Faculty of Engineering and IT, Australian National University, Canberra ACT 0200, Australia

Stefan W. Glunz

Fraunhofer Institute for Solar Energy Systems (ISE), Heidenhofstrasse 2, 79110 Freiburg, Germany

Richard R. King

Spectrolab Inc., 12500 Gladstone Avenue, Sylmar, California 91342

\author{
Gernot Heiser ${ }^{\mathrm{a})}$ \\ Centre for Photovoltaic Engineering, University of New South Wales and School of Computer Science \\ and Engineering, University of New South Wales, Sydney, 2052, Australia
}

Andreas Schenk

Integrated Systems Laboratory, ETH Zurich, Gloriastrasse 35, 8092 Zurich, Switzerland

(Received 25 February 2002; accepted for publication 28 June 2002)

\begin{abstract}
We have established a simulation model for phosphorus-doped silicon emitters using Fermi-Dirac statistics. Our model is based on a set of independently measured material parameters and on quantum mechanical calculations. In contrast to commonly applied models, which use Boltzmann statistics and apparent band-gap narrowing data, we use Fermi-Dirac statistics and theoretically derived band shifts, and therefore we account for the degeneracy effects on a physically sounder basis. This leads to unprecedented consistency and precision even at very high dopant densities. We also derive the hole surface recombination velocity parameter $S_{p o}$ by applying our model to a broad range of measurements of the emitter saturation current density. Despite small differences in oxide quality among various laboratories, $S_{p o}$ generally increases for all of them in a very similar manner at high surface doping densities $N_{\text {surf }}$. Pyramidal texturing generally increases $S_{p o}$ by a factor of five. The frequently used forming gas anneal lowers $S_{p o}$ mainly in low-doped emitters, while an aluminum anneal (Al deposit followed by a heat cycle) lowers $S_{p o}$ at all $N_{\text {surf }}$. () 2002 American Institute of Physics. [DOI: 10.1063/1.1501743]
\end{abstract}

\section{PURPOSE OF THIS WORK}

The electronics industry has, for some time, been using advanced numerical modeling for developing and optimizing devices very successfully. In contrast, one of its fastest growing branches, solar cell manufacturing, uses computer simulations only sparingly. This is partly so because common simulation techniques reproduce two of the most important device regions inadequately. First, many types of commercially manufactured silicon cells contain highly doped device regions that are performance limiting: an $n^{+}$emitter and a $p^{+}$backsurface field. These regions are commonly modeled with Boltzmann statistics, although Fermi-Dirac statistics should be applied instead, because Pauli-blocking reduces the $p$ - $n$ product significantly. To compensate for this lack, theories have been developed that use apparent band-gap narrowing or other effective parameters, although it has been shown on mathematically rigorous grounds that such adjust-

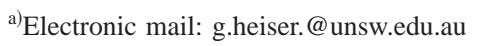

ments lead to various inconsistencies. ${ }^{1,2}$ Hence, common simulations adjust the quasi-Fermi level of minority carriers only imprecisely in such device parts. ${ }^{1-4}$ Second, the backsurface field of many commercial cells is aluminum doped but is modeled with the intrinsic density-of-states. Aluminum forms an impurity band which is particularly far away from the valence band edge. Neglecting this leads to the wrong adjustments of the (quasi-) Fermi level of majority carriers. Hence, by using Fermi-Dirac statistics, the model presented here makes modeling a more powerful tool for the development of many types of commercial solar cells. This article deals mainly with the first issue, while the second issue is partly dealt with in a separate article. ${ }^{5}$

When comparing a simulation model with an experiment, one needs to keep in mind that only the total losses in the emitter can be measured via the emitter saturation current-density $J_{o e}$. This makes quantifying the recombination velocity parameter $S_{p o}$ at the surface of the emitter par- 
ticularly challenging: $S_{p o}$ needs to be separated from losses occurring in the bulk of the emitter, and this separation has been achieved only with theoretical models. As different models divide the losses in the bulk and at the surface into different proportions, the resulting $S_{p o}$ is model dependent. This restricted approach has been used steadily, although surface recombination losses limit the efficiency in many types of solar cells.

In the past, various models have been used to extract $S_{p o}$ from $J_{o e}$. As outlined above, the commonly applied models use effective parameters to compensate for the incorrect statistics. In this article, however, we establish a parameter set for the use of Fermi-Dirac statistics, and we reevaluate the $J_{o e}$ measurements made by Cuevas et al. ${ }^{6-8}$ Glunz et al. ${ }^{9}$ Kerr et al. ${ }^{10,11}$ and King et al. ${ }^{12}$ We apply the band-gap narrowing model recently developed by Schenk ${ }^{13}$ on quantum mechanical principles, and use the silicon parameter-set established by Altermatt et al. ${ }^{14-25}$ Preliminary results of this project were published in Refs. 24 and 26.

\section{FERMI-DIRAC STATISTICS}

Most published calculations for solar cells are based on Boltzmann statistics, although it was established in the early days of semiconductor physics that Fermi-Dirac statistics need to be applied for dopant densities $N_{\mathrm{dop}} \geqslant 1$ $\times 10^{19} \mathrm{~cm}^{-3}$. Boltzmann statistics are popular because the performance-limiting parts of high-efficiency cells have been doped lower than $1 \times 10^{19} \mathrm{~cm}^{-3}$, and Fermi-Dirac (FD) statistics are cumbersome to include in analytical models. An outcome of this is that physical parameters, such as band-gap narrowing, have been extracted from experiments using Boltzmann statistics as well. This enforced the subsequent use of Boltzmann statistics, because such a parameter set partly compensated for the incorrect statistics. However, many commercial solar cells contain performance-limiting regions with $N_{\text {dop }} \gtrsim 1 \times 10^{19} \mathrm{~cm}^{-3}$, where Pauli-blocking becomes significant. We therefore use FD statistics, which can be applied in numerical modeling without difficulties. This makes it necessary to adapt those parameters to FD statistics that have been commonly extracted from experiments using Boltzmann statistics.

In analogy to the law of mass action for ideal gases, the well-known relation $n_{i}^{2}=p n$ between the intrinsic carrier density $n_{i}$ and the electron and hole densities, $n$ and $p$, holds only if the carriers do not interact strongly with each other, i.e., in intrinsic and moderately doped silicon. In heavily doped $n$-type silicon, we calculate $n$ using

$$
n=N_{c} F_{1 / 2}\left(\frac{E_{f n}-E_{c}^{(0)}+\Delta E_{c}}{k T}\right),
$$

where $N_{c}$ is the effective density of states in the conduction band, $F_{1 / 2}$ the Fermi integral of order $1 / 2, E_{f n}$ the quasiFermi level for electrons, $E_{c}^{(0)}$ the energy of the intrinsic conduction band edge, $\Delta E_{c}$ is the shift of the conduction band edge due to BGN, $k$ is Boltzmann's constant, and $T$ is the absolute temperature. We can express $p$ in an analogous way. However, since the holes are non-degenerate in $n$-type emitters, the simple Boltzmann expression for $p$ is appropriate:

$$
p=N_{v} \exp \left(-\frac{E_{f p}-E_{v}^{(0)}-\Delta E_{v}}{k T}\right),
$$

where the symbols have equivalent meaning as in Eq. (1) but for holes and the valence band. In order to clarify the influence of FD statistics on the simulated $J_{o e}$, we write the $p n$ product in such a way that $n_{i}^{2}$, degeneracy, band-gap narrowing, and deviations from thermal equilibrium are separated in different factors:

$$
\begin{aligned}
p n= & N_{c} N_{v} \exp \left(-\frac{E_{c}^{(0)}-E_{v}^{(0)}}{k T}\right) \\
& \times F_{1 / 2}\left(\frac{E_{f n}-E_{c}^{(0)}+\Delta E_{c}}{k T}\right) \\
& \times \exp \left(-\frac{E_{f p}-E_{v}^{(0)}-\Delta E_{v}}{k T}\right) \exp \left(\frac{E_{c}^{(0)}-E_{v}^{(0)}}{k T}\right) \\
= & n_{i}^{2} \frac{F_{1 / 2}\left(\frac{E_{f n}-E_{c}^{(0)}}{k T}\right)}{} \\
& \exp \left(-\frac{E_{c}^{(0)}-E_{f n}}{k T}\right) \\
& \times \frac{F_{1 / 2}\left(\frac{E_{f n}-E_{c}^{(0)}+\Delta E_{c}}{k T}\right)}{F_{1 / 2}\left(\frac{E_{f n}-E_{c}^{(0)}}{k T}\right)} \exp \left(\frac{\Delta E_{v}}{k T}\right) \exp \left(\frac{E_{f n}-E_{f p}}{k T}\right) \\
\equiv & n_{i}^{2} \times \gamma_{\mathrm{deg}} \times \gamma_{\mathrm{BGN}} \times \gamma_{\text {neq }} .
\end{aligned}
$$

The degeneracy factor $\gamma_{\text {deg }}$ is a measure of how much the $p n$ product deviates from its value as an ideal gas. Only if the electrons are also nondegenerate, as is the case in low-doped $n$-type emitters, can we have $\gamma_{\mathrm{deg}} \rightarrow 1$ and $\gamma_{\mathrm{BGN}} \rightarrow 1$, so that $p n \rightarrow n_{i, \text { eff }}^{2}=n_{i}^{2} \exp \left[\left(\Delta E_{c}+\Delta E_{v}\right) / k T\right]$, i.e., only then do Boltzmann statistics describe the situation well. To illustrate the difference between Boltzmann and FD statistics, we plot Eq. (3) in Fig. 1, as simulated in a highly doped emitter. We use FD statistics with BGN of Ref. 13, or Boltzmann statistics with BGN of Ref. 8, respectively (see below for details of these simulations). While BGN increases the $p n$ product towards the surface of this diffused emitter, degeneracy tends to decrease it, leading to a maximum value of $p n$ within the bulk. Such counteracting effects between BGN and carrier degeneracy cannot be adequately quantified using Boltzmann statistics and apparent BGN $\Delta E_{g}^{\text {app }}{ }^{1,2}$ For example, since Boltzmann statistics overestimate $p, \Delta E_{g}^{\text {app }}$ is made smaller than the actual shifts of the band edges to adjust the $p n$ product, i.e., $\Delta E_{g}^{\mathrm{app}}<\Delta E_{g} \equiv \Delta E_{c}+\Delta E_{v}$. This is apparent, for example, in Fig. 7 of Ref. 11. This correction, in turn, lowers the band bending across the $p$ - $n$ junction of the device and hence the electric field. For further discussions of this topic, see Refs. 1, 2, 24, and 27. 


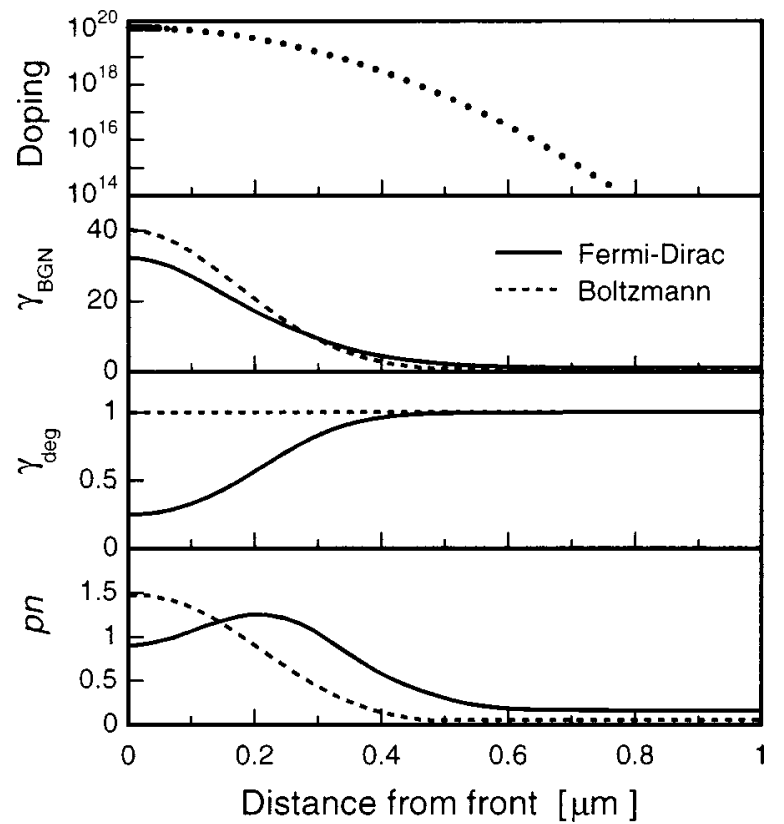

FIG. 1. Simulation of a heavily doped emitter (M4X of Ref. 12) with Boltzmann and with Fermi-Dirac statistics, respectively. Shown are the phosphorus dopant profile in units of $\mathrm{cm}^{-3}$, band-gap narrowing $\gamma_{\mathrm{BGN}}$, degeneracy $\gamma_{\mathrm{deg}}$, and the $p n$ product in units of $10^{33} \mathrm{~cm}^{-6}$, all using Eq. (3).

\section{EXPERIMENTAL FOUNDATION OF THE MODEL}

In the simulation of emitters, many relevant silicon parameters and device models come into play. These are $n_{i}$, BGN, Auger recombination, the minority carrier mobility, the density of states (DOS) affected by doping, and the incomplete ionization of dopants. Apart from $n_{i}$, all of these parameters and models describe effects caused by carriercarrier and carrier-dopant interactions. Accordingly, we can regard the improvements, made in the understanding of the highly doped $\mathrm{Si}$ over the past few years, as a shift from the ideal-gas model to the many-body theory. This shift needs to be experimentally substantiated, as is done in the following.

\section{A. New intrinsic carrier density of silicon}

The intrinsic carrier density is of fundamental importance since it enters into almost all calculations that relate device responses to excitations. For example, $n_{i}$ strongly influences the minority carrier densities in low-doped emitters, and hence determines their $J_{o e}$. Prior to $1990, n_{i}=1.45$ $\times 10^{10} \mathrm{~cm}^{-3}$ was commonly used in crystalline silicon at $T$ $=300 \mathrm{~K}$, leading to significant deviations between the theoretically predicted and the measured behavior of devices. ${ }^{28}$ In 1991, Green and Sproul measured $n_{i}=1.00$ $\times 10^{10} \mathrm{~cm}^{-3},{ }^{29,30}$ which is significantly lower than the previously used value. This is the most widely accepted value of $n_{i}$ in the silicon community. However, it has been recently shown ${ }^{18,21}$ that the measurements of Sproul and Green were influenced by BGN, even though the dopant density of their samples was low. These recent investigations showed that $n_{i}$ is slightly lower, namely $n_{i}=9.65 \times 10^{9} \mathrm{~cm}^{-3}$, which is the asymptotic value towards very low dopant densities in Fig. 2. These investigations also resolved a long prevailing discrepancy with the measurements of Misiakos and Tsamakis. ${ }^{31}$

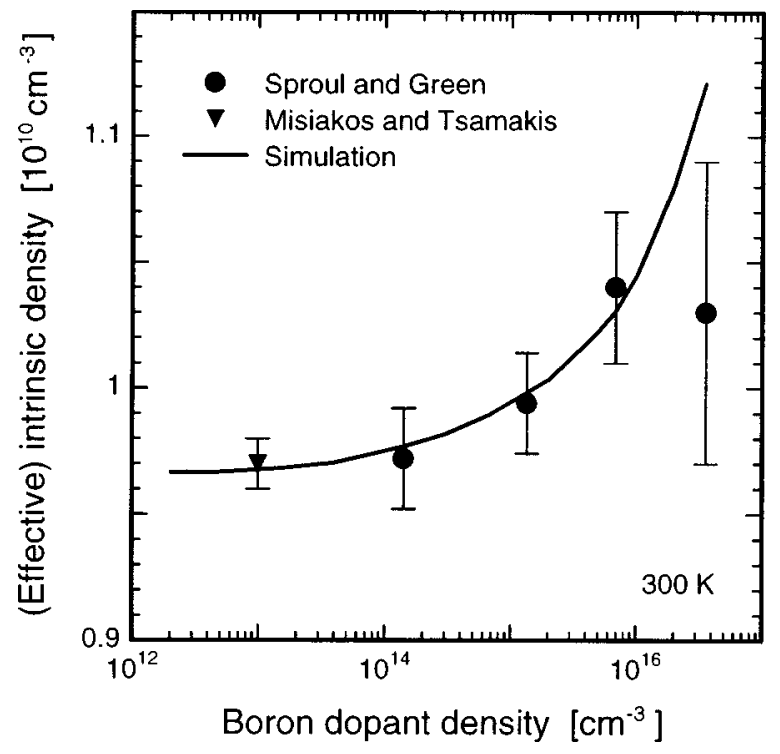

FIG. 2. (Effective) intrinsic carrier density of crystalline silicon, as a function of boron dopant density, determined by Sproul and Green (see Refs. 29 and 30) using their analytical model (circles), the value measured by Misiakos and Tsamakis (see Ref. 31) (triangle), and our simulations using $n_{i}$ $=9.65 \times 10^{9} \mathrm{~cm}^{-3}$ and the band-gap narrowing model of Ref. 13 (solid line).

The important point of this article is that these recent investigations made use of a BGN model that is based on the many-body theory, where both the exchange-correlation selfenergy of the free carriers and the correlation energy of the carrier-dopant interaction were treated on an equal basis. ${ }^{13}$ Hence, we use $n_{i}=9.65 \times 10^{9} \mathrm{~cm}^{-3}$ in this study.

King et al. ${ }^{12}$ applied the old $n_{i}=1.45 \times 10^{10} \mathrm{~cm}^{-3}$ for measuring $J_{o e}$ and calculating $S$. Their data evaluation was revised by Cuevas et al. ${ }^{6,8}$ with Sproul and Green's $n_{i}$ value. Kerr et al. ${ }^{11}$ and Glunz et al. ${ }^{9}$ applied Sproul and Green's $n_{i}$ value as well. The changes in $S$, caused by using the new $n_{i}=9.65 \times 10^{9} \mathrm{~cm}^{-3}$ instead of Sproul and Green's value, are in most cases minor and smaller than the uncertainties imposed by the limited precision of the $J_{o e}$ measurements.

\section{B. Apparent versus theoretically derived band-gap narrowing}

Among the material parameters and models, it has mainly been BGN that has been manipulated to compensate for the discrepancies caused by Boltzmann statistics; so we mainly need to reassess BGN when using FD statistics.

So far, solar cells have been mostly simulated with empirical BGN models that were derived from electronic measurements ${ }^{25,32-43}$ of highly doped silicon, as is shown in Fig. 3. Absorption ${ }^{44-47}$ and most other optical methods ${ }^{48-51}$ yield considerably lower BGN values, as shown by the open symbols. The electronically measured apparent BGN values were extracted from transport measurements, and were influenced by the transport model involved in the data evaluation. In particular, they depended on $n_{i}$ and the mobility of minority carriers $\mu_{\min }$. For example, del Alamo and Swanson ${ }^{42}$ revised the BGN values of Fig. 3(c) mainly by adjusting the 

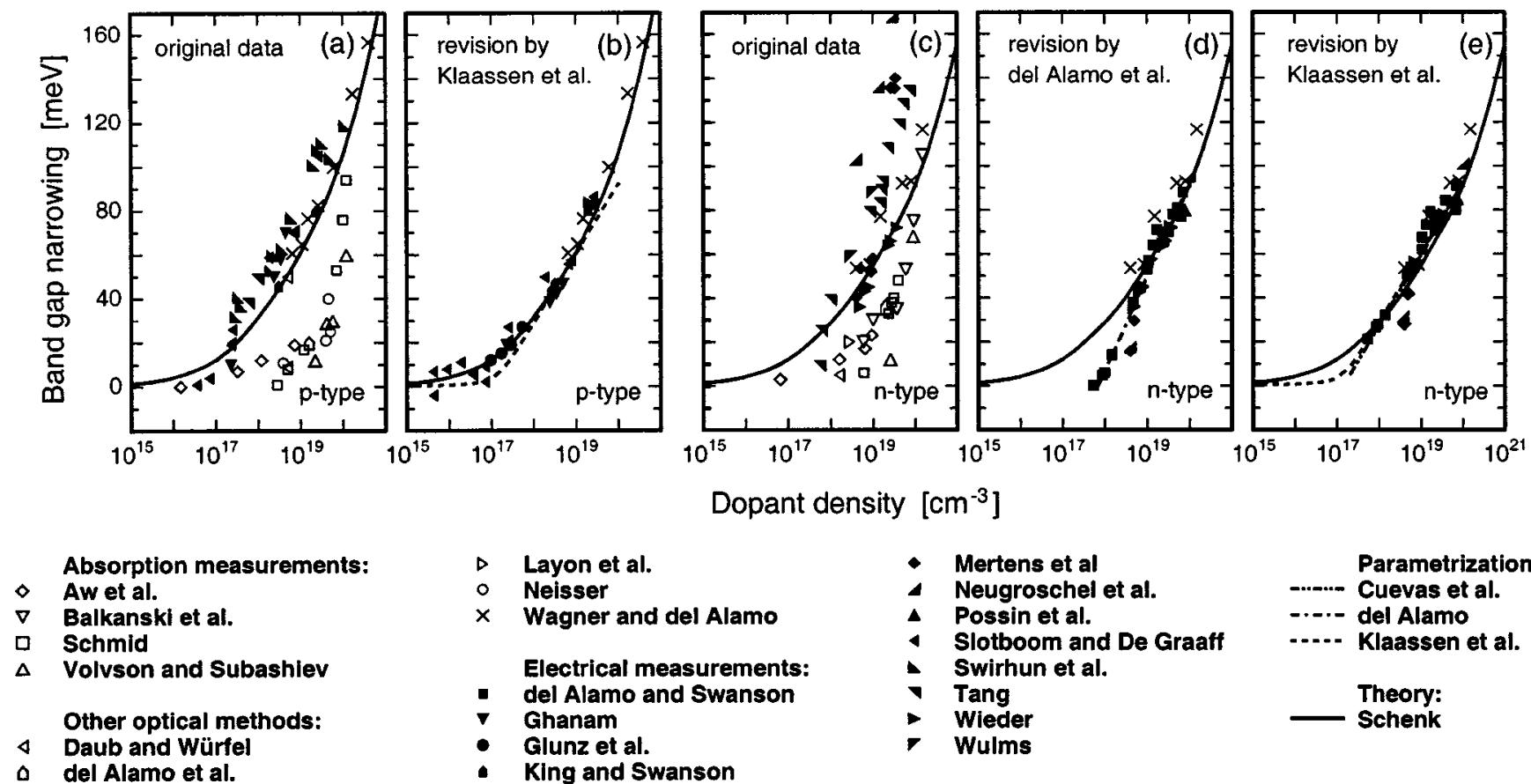

\begin{tabular}{ll} 
Layon et al. \\
$\times \quad$ Neisser \\
$\times \quad$ Wagner and del Alamo \\
Electrical measurements: \\
- del Alamo and Swanson \\
- Ghanam \\
- Klunz et al. \\
\hline
\end{tabular}
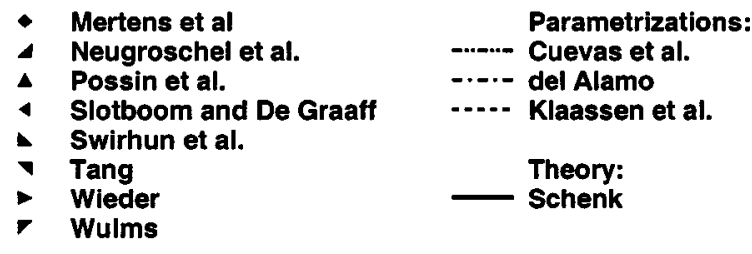

FIG. 3. Band-gap narrowing in $n$ - and $p$-type silicon, measured electronically (Refs. 25, 32-43) (filled symbols), by absorption (Refs. 44-47) and other optical methods (Refs. 48-51) (open symbols), and by photoluminescence (crosses) (Ref. 52). Shown are the original data, a revision and parameterization made by del Alamo and Swanson (Ref. 42) and a revision and parameterization by Klaassen et al. (Ref. 32) The recent calculations (Ref. 13) (solid lines) are not apparent BGN data and therefore can only be compared with the measurements with care.

involved $\mu_{\min }$ to improved experimental results, and they obtained Fig. 3(d). However, they used the old value of $n_{i}$, so Klaassen et al. ${ }^{32}$ revised the BGN values of Fig. 3(d) one time further by adjusting $n_{i}$ to a lower value, with the outcome of Fig. 3(e). When $n_{i}$ was adjusted to a smaller value, BGN increased at low dopant densities, because a too-high $n_{i}$ value had led to compensation by a too-small BGN value. This is even more apparent in p-type silicon, as shown in Figs. 3(a) and 3(b). A reevaluation of the BGN data with $n_{i}$ $=9.65 \times 10^{9} \mathrm{~cm}^{-3}$ would shift the values in Klaassen's collection towards higher values by less than the symbol size.

Independently of these modifications, the important points here are that:

(i) These BGN values do not reflect the actual band-gap shrinkage $\Delta E_{g}$, but are a conglomerate of various effects, including degeneracy ${ }^{3,4,11}$ at high doping levels, the change in DOS due to the formation of an impurity band ${ }^{2}$ at medium to high dopant densities, and asymmetry in gap shrinkage. ${ }^{1,2,4}$

(ii) Since degeneracy effects are partly compensated for in $\Delta E_{g}^{\text {app }}$, we cannot apply Fermi-Dirac statistics together with $\Delta E_{g}^{\text {app }}$ values, as this would overestimate the degeneracy effects.

(iii) Since the $\Delta E_{g}^{\text {app }}$ values shown in Fig. 3 were obtained using various transport models, it is not obvious exactly how degeneracy influences $\Delta E_{g}^{\text {app }}$, and we cannot correct the $\Delta E_{g}^{\text {app }}$ values for FD statistics with one single and well-recognized procedure.

Instead, we choose to base our simulations on theoretically derived models, such as the comprehensive BGN model of Ref. 13. This BGN model was recently derived from a non-self-consistent, full random phase approximation (RPA) formalism at finite temperatures, where both carriercarrier and carrier-dopant interactions were treated on an equal basis. The dispersive quasiparticle shift in RPA quality was numerically calculated and, based on these numerical results, Padé approximations of the band edge energies $\left(\Delta E_{c}\right.$ and $\Delta E_{v}$ ) were constructed in terms of carrier densities, dopant densities, and temperature. Since this model provides the shift of the band edge energies, it can be used together with FD statistics.

As this approach differs considerably from the determination of $\Delta E_{g}^{\mathrm{app}}$, we must be careful when comparing the model with $\Delta E_{g}^{\text {app }}$ in Fig. 3. At low dopant densities and under low-level injection conditions, both carrier types are nondegenerate, and the DOS is essentially ideal. Reference 4 also shows that, under such conditions, the effects of asymmetry in BGN $\left(\Delta E_{c} \neq \Delta E_{v}\right)$ are unimportant. Hence, we can directly compare the model with $\Delta E_{g}^{\text {app }}$ under these conditions, and Figs. 3(b) and 3(e) show that there is good agreement between the two approaches. These figures also show that, compared to photoluminescence measurements ${ }^{52}$ (shown as crosses), the model provides a similar but slightly larger $\Delta E_{g}$ (up to $10 \%$ ) in the high doping range. This is expected because the band tails are neglected in the rigidshift model of Ref. 13 (the approximations made in the model are discussed elsewhere ${ }^{21}$ in more detail). However, the model is a good approximation, because band tails host immobile carriers, while $n$ and $p$ enter the basic semiconductor equations ${ }^{53}$ either through the Poisson equation as charge carriers that are not electrostatically compensated, or through 


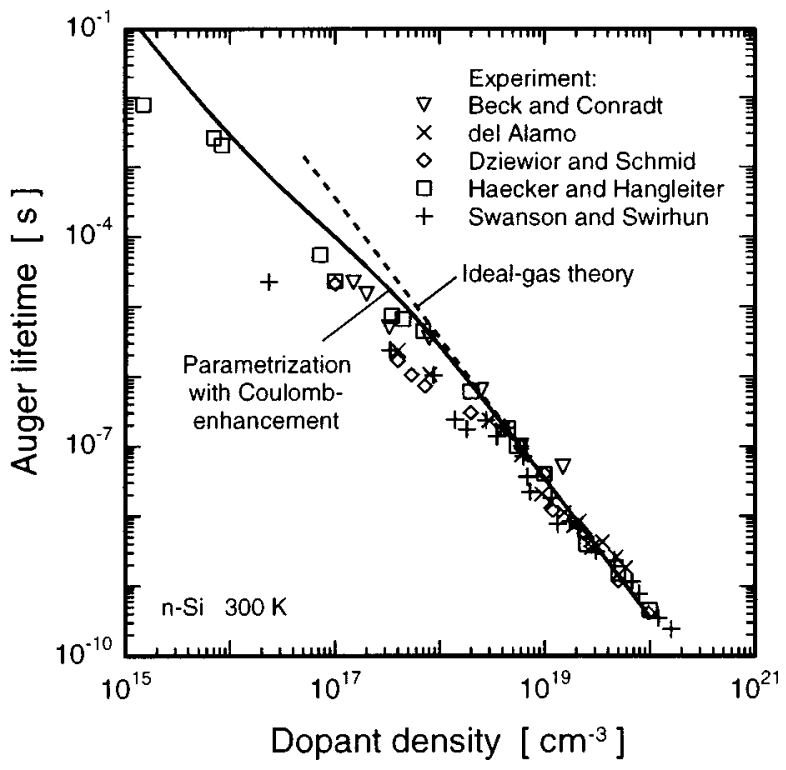

FIG. 4. Measured Auger lifetimes (Refs. 42, 54-57) at low-injection conditions, as a function of dopant density $N_{\text {dop }}$ in $n$-type silicon. At $N_{\text {dop }}$ $\lesssim 10^{18} \mathrm{~cm}^{-3}$, the lifetimes are lower than the ideal-gas limit (dashed lines) due to Coulomb enhancement (Refs. 17, 19, 59) (solid line).

the continuity equations accounting for currents, i.e. for entirely mobile carriers. Hence, the majority carriers in immobile tail states are not expected to contribute considerably to BGN relevant to electronic devices. Similar arguments apply to the donor band at medium dopant densities. In Sec. V A we will demonstrate that the usage of FD statistics and the BGN model of Ref. 13 give consistent results.

\section{Recombination losses}

In many types of emitters, the dominating recombination loss occurs through the band-to-band Auger process, which is an intrinsic property of silicon. Figure 4 shows that at dopant densities $N_{\text {dop }} \geqslant 1 \times 10^{18} \mathrm{~cm}^{-3}$, the measured Auger recombination lifetime ${ }^{42,54-57} \tau_{A}$ can be well reproduced assuming noninteracting free particles, i.e., by $\tau_{A}$ $=1 /\left(C_{n} N_{\text {dop }}^{2}\right)$ (see dashed line). At these dopant densities, we use the Auger coefficient $C_{n}=2.8 \times 10^{-31} \mathrm{~cm}^{6} \mathrm{~s}^{-1}$, as reported by Dziewior and Schmid, ${ }^{55}$ because their data show the smallest scatter of all the published lifetime data used to determine $C_{n} \cdot{ }^{17}$ In their extraction of $S$, Cuevas et al. chose a slightly different $C_{n}$ value, while King et al. also used Dziewior and Schmid's value in the emitter.

The Auger lifetime is enhanced by Coulomb interactions at $N_{\text {dop }} \leqslant 1 \times 10^{18} \mathrm{~cm}^{-3} \cdot{ }^{17,58}$ A few different parameterizations of this enhancement have been published. ${ }^{17,19,59}$ However, we will show that this enhancement influences the simulations of emitters only marginally, and it is therefore irrelevant which of the parameterizations is chosen in our simulations.

The other recombination loss is surface recombination. At the dopant densities found in emitters, it is well quantified by the Shockley-Read-Hall theory: ${ }^{60,61}$

$$
U_{s}=\frac{n_{s} p_{s}-n_{i, \mathrm{eff}}^{2}}{S_{p o}^{-1}\left(n_{s}+n_{1}\right)+S_{n o}^{-1}\left(p_{s}+p_{1}\right)},
$$

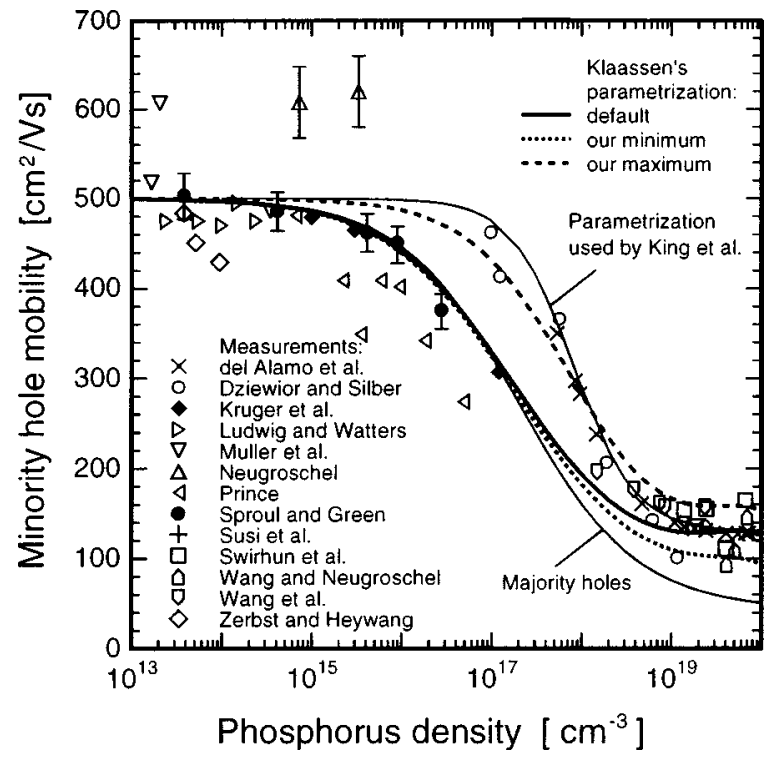

FIG. 5. Minority-carrier hole mobility $\mu_{h \text {,min }}$, measured by various authors, (Refs. 35, 63-76) and parametrized by Klaassen (Ref. 77). A parameterization used in the work of King et al., (Ref. 12) and a parameterization of the majority-carrier hole mobility (Ref. 93) are shown as well.

where $n_{s}$ and $p_{s}$ are the electron and hole densities at the surface, which may be influenced by the surface charge density. The parameters

$$
n_{1}=n_{i, \text { eff }} e^{\left(E_{t}-E_{i}\right) / k T} \quad p_{1}=n_{i, \text { eff }}^{2} / n_{1}
$$

relate the energy level of the defect $E_{t}$ to the intrinsic energy level $E_{i}$. The recombination rate $U_{s}$ is limited by holes in $n$-type emitters, i.e., by the recombination velocity parameter for holes $S_{p o}$. We assume in our simulations that the bulk SRH lifetime $\tau_{\mathrm{SRH}}=2 \mathrm{~ms}$, which is a reasonable value for high-purity float-zone material, and is independent of $N_{\text {dop }} \cdot{ }^{17}$ The choice of $\tau_{\mathrm{SRH}}$ does not affect the simulations presented here.

\section{Minority carrier mobility}

At dopant densities found in emitters, the mobility of minority holes $\mu_{h \text {,min }}$ is limited by hole-dopant interactions in a complex manner and is not well understood. ${ }^{62}$ It is expected that at $N_{\mathrm{dop}} \gtrsim 10^{17} \mathrm{~cm}^{-3}, \mu_{h, \text { min }}$ is higher than the majority-carrier hole mobility $\mu_{h \text {,maj }}$, because scattering by the positively charged phosphorus ions is less effective than by the negatively charged boron ions. This effect is experimentally and theoretically well established in the case of electron mobility. ${ }^{22}$ However, in the case of hole mobility, the measured values spread significantly (see symbols in Fig. 5) ${ }^{35,63-76}$ In the low dopant density range, the most reliable data are the photoconductance decay measurements of Sproul et al. ${ }^{70}$ and the recent Shockley-Haynes measurements of Kruger et al. ${ }^{65}$ because their method yields $\mu_{h \text {,min }}$ rather directly, while most other methods are more indirect. The $\mu_{h, \min }$ values given by del Alamo et al. and by Dziewior and Silber are significantly higher than theoretically expected in the range $N_{\text {dop }}=10^{17}-10^{18} \mathrm{~cm}^{-3}$, and are questionable. King et al. relied on these values in the data evaluation of their $J_{o e}$ measurements; we show their parameterization in 
Fig. 5 as well. Also shown for comparison is a parameterization of the majority hole mobility by Masetti, Severi, and Solmi. ${ }^{93}$ Most important to emitter $J_{o e}$ simulations is $\mu_{h, \text { min }}$ at $N_{\text {dop }}>10^{18} \mathrm{~cm}^{-3}$. The measurements scatter by $\pm 40 \%$ in this range. We use the default setting of Klaassen's parameterization, ${ }^{77}$ as shown in Fig. 5. Cuevas et al. used a very similar model. To estimate the maximum and minimum error bounds of $\mu_{h \text {,min }}$ on $J_{o e}$, we have adjusted Klaassen's parameterization in some cases, as shown by the dashed lines.

The mobility affects our $J_{o e}$ simulations significantly only in some dopant profiles, where the emitter is at the verge of being transparent; ${ }^{78}$ i.e., where all carriers injected from the $p-n$ junction may just reach the surface. In such cases, the error bounds of $\mu_{h \text {,min }}$ determine how much surface recombination affects $J_{o e}$, so these error bounds provoke the largest uncertainties in our simulations. In the other emitters, where the injected carriers reach the surface easily, or cannot reach the surface at all (i.e., where there is a dead layer), $\mu_{h, \min }$ does not influence $J_{o e}$ significantly; the uncertainties of our simulations are then induced by the error bounds related to the $J_{o e}$ measurements.

\section{E. Density-of-states at high doping levels}

It is common in simulations to use the intrinsic densityof-states (DOS) at all dopant densities. At high $N_{\text {dop }}$, however, the dopants form a band near the band edge, which shifts the Fermi level further away from the band edge than when using the intrinsic DOS at all dopant densities. ${ }^{5}$ As $E_{f n}$ is situated close to this donor band, the donor states are considerably occupied by electrons so that $n<N_{\text {dop }}$, which is called incomplete ionization. ${ }^{5,22}$ As these effects are biggest near the Mott transition (which occurs at $N_{\text {dop }}=3.74$ $\times 10^{18} \mathrm{~cm}^{-3}$ ), they affect only lightly doped emitters. Since the donor band is rather close to the conduction band in Si:P, the effects are considerably weaker than in $p$-type Si:Al. Hence, these effects are minor compared to the error bounds of the $J_{o e}$ measurements. We include the donor band in some lightly doped emitters in Sec. VB to demonstrate these effects.

At very high $N_{\text {dop }}$, where the donor band has merged with the conduction band, both band edges are modified by tail states, which arise due to disorder. ${ }^{79}$ Deep tail states are localized, while the shallow ones are a hybridization between localized and extended states, ${ }^{80,81}$ and therefore they host immobile carriers. The tail state density is too small to cause a considerable amount of incomplete ionization. ${ }^{5}$ In contrast, the valence band edge hosts only a small number of holes, so that tailing effects may be more noticeable. Pan et al. ${ }^{82}$ predicted that the equilibrium hole density will increase by a factor of two due to band tails. Unfortunately, they fitted their tail density to room-temperature photoluminescence (PL) measurements, ${ }^{83}$ neglecting effects that cause the broadening of the PL lines. ${ }^{79,84,85}$ For these reasons, tailing affects the minority-carrier transport considerably less than proposed by Pan et al. Due to the lack of experimental data, we are unable to quantify the influence of the minority-band tailing on our $J_{o e}$ simulations.

\section{SIMULATION TECHNIQUE}

We use the device simulator Dessis ${ }^{86}$ which—in contrast to some analytical models of previous studies-solves the well-known, fully coupled set of semiconductor differential equations ${ }^{53}$ numerically and in a self-consistent way. Great care has been taken that the numerical errors stay within negligible boundaries. We apply FD statistics at $300 \mathrm{~K}$, together with the models and parameters of Sec. III. The $n^{+} \mathrm{pn}^{+}$test structures were measured in Refs. $6-12$ by photoconductance transient decay and quasi-steady-state photoconductance. We find it unnecessary to use transient models in our simulations, because we can draw on the general definition $^{24}$ of $J_{o e}$,

$$
J_{o e} \equiv \frac{J_{n}\left(x_{e}\right)}{n\left(x_{e}\right) p\left(x_{e}\right)-n_{i, \mathrm{eff}}^{2}\left(x_{e}\right)} n_{i, \mathrm{eff}}^{2}\left(x_{e}\right),
$$

and hence need to simulate the devices only in steady-state open-circuit condition. Equation (6) shows that $J_{o e}$ is related to the electron recombination current $J_{n}$ across the $p$ - $n$ junction, and to the excess $p n$ product at the edge $x_{e}$ of the space charge region. We employ the effective carrier density $n_{i, \text { eff }}$ instead of $n_{i}$, to account for any BGN that may be induced by the injected carriers at high-injection conditions. The BGN model of Ref. 13 accounts for carrier-induced BGN. ${ }^{20}$

In the experiment, most of the diode structures had no metal contacts. A numerical simulation requires at least one "contact" in order to provide a zero level for the electrostatic potential. We therefore attach a very small floating gate at the edge of our 2D domain. This gate does not influence the recombination properties in the silicon. As doping profiles, we took the SIMS or stripping-Hall data of Refs. 6-12. According to Ref. 87, the SIMS profiles represent the electrically active phosphorus density in all the emitters used in this study.

\section{RESULTS}

\section{A. Verification of consistency}

While we are able to base our models of BGN, Auger recombination and mobility on independent measurements, there is no method known to us that allows us to independently measure the surface recombination velocity parameter of holes, $S_{p o}$. Hence, we must generally treat $S_{p o}$ as unknown. An exception is when $J_{o e}$ is measured on emitters covered with a thin metal layer: ${ }^{8} S_{p o}$ of such surfaces is then limited by the thermal velocity of free carriers $v_{t h}$, i.e., $S_{p o}=1.562 \times 10^{7}$ and $S_{n o}=2.042 \times 10^{7} \mathrm{~cm} / \mathrm{s}$ at $300 \mathrm{~K}$, independently of the crystal orientation. ${ }^{86,88,89}$ Hence, these metal-coated samples give us the precious opportunity to verify the consistency of our applied models and material parameters.

Indeed, we are able to reproduce the measured $J_{o e}$ values of all metal-coated emitters (i.e., at $N_{\text {dop }}<2$ $\times 10^{20} \mathrm{~cm}^{-3}$ ) without any further adjustments. This is a crucial result. It demonstrates that our model is consistent, and that this study is more than another adjustment of parameters to extract $S_{p o}$ from $J_{o e}$ measurements: it also introduces the 


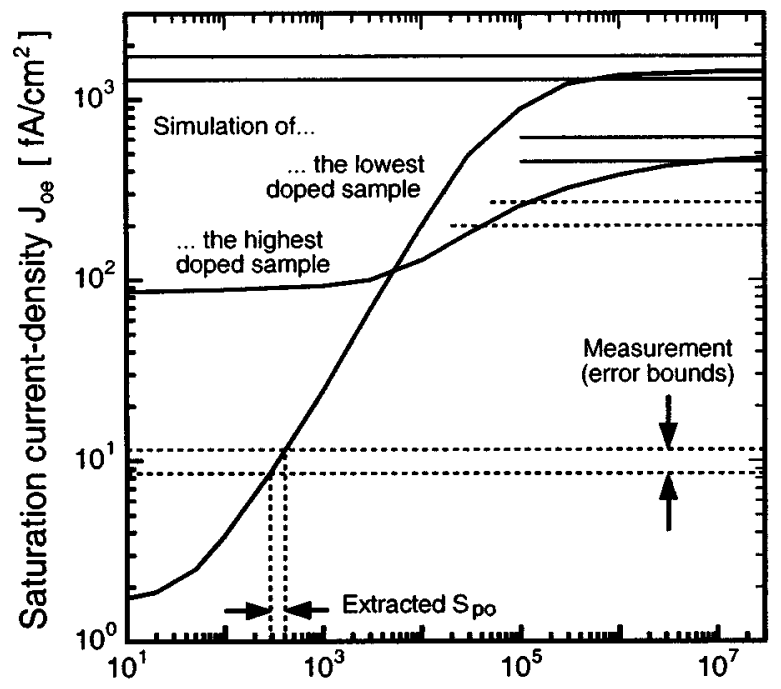

Surface recombination velocity $S_{p o}[\mathrm{~cm} / \mathrm{s}$ ]

FIG. 6. Simulated saturation current-density $J_{o e}$ of the highest and lowest doped emitter of Cuevas et al. (Refs. 6-8) with $N_{\text {peak }}=2.2 \times 10^{18}$ and 2 $\times 10^{20} \mathrm{~cm}^{-3}$, respectively. The error bounds of the measured $J_{o e}$ of metalcoated and oxide-passivated surfaces are indicated by the horizontal solid and dashed lines, respectively.

physically sound statistics. With previous models, such consistency has not been achieved at high $N_{\text {dop }}$, mainly because Boltzmann statistics were applied.

Figure 6 shows the simulated $J_{o e}$ of both the lowest and the highest doped emitter in the collection of Cuevas et al., where the doping density at the surface $N_{\text {surf }}$ is $2.2 \times 10^{18}$ and $2 \times 10^{20} \mathrm{~cm}^{-3}$, respectively. The simulated $J_{o e}$ approaches the measured values (horizontal solid lines) when $S_{p o}$ approaches $v_{t h}$. With Boltzmann statistics, $J_{o e}$ would overshoot the measured values in the highly doped emitter. Generally, the simulated $J_{o e}$ depends with varying sensitivity on $S_{p o}$. For example, the bulk losses are large in the highly doped sample and dominate $J_{o e}$ at $S_{p o}<1000 \mathrm{~cm} / \mathrm{s}$, so the simulated $J_{o e}$ curve flattens out towards low $S_{p o}$. Towards high $S_{p o}$ values, the surface recombination rate becomes limited by the hole current injected from the $p$ - $n$ junction, hence $J_{o e}$ flattens out again.

\section{B. Extraction of the surface recombination velocity}

After having verified its consistency, we use our model to extract $S_{p o}$ from the $J_{o e}$ measurements; $S_{p o}$ is an important material parameter in devices with passivated surfaces. Figure 6 indicates that $J_{o e}$ is far more sensitive to $S_{p o}$ in a low doped than in a highly doped emitter (of comparable junction depth). Accordingly, we are able to extract $S_{p o}$ more precisely in some emitters than in others. For example, Fig. 6 also shows the measured $J_{o e}$ values of devices with oxidepassivated surfaces (dashed lines). The simulated $J_{o e}$ drops more steeply near the measured $J_{o e}$ value in the low-doped emitter, and hence the error bounds of the extracted $S_{p o}$ are smaller than in the case of the highly doped emitter. In some cases, the error bounds of $\mu_{h, \text { min }}$ need to be added to these error bounds.

Figure 7 shows the extracted $S_{p o}$ as a function of $N_{\text {surf }}$ of

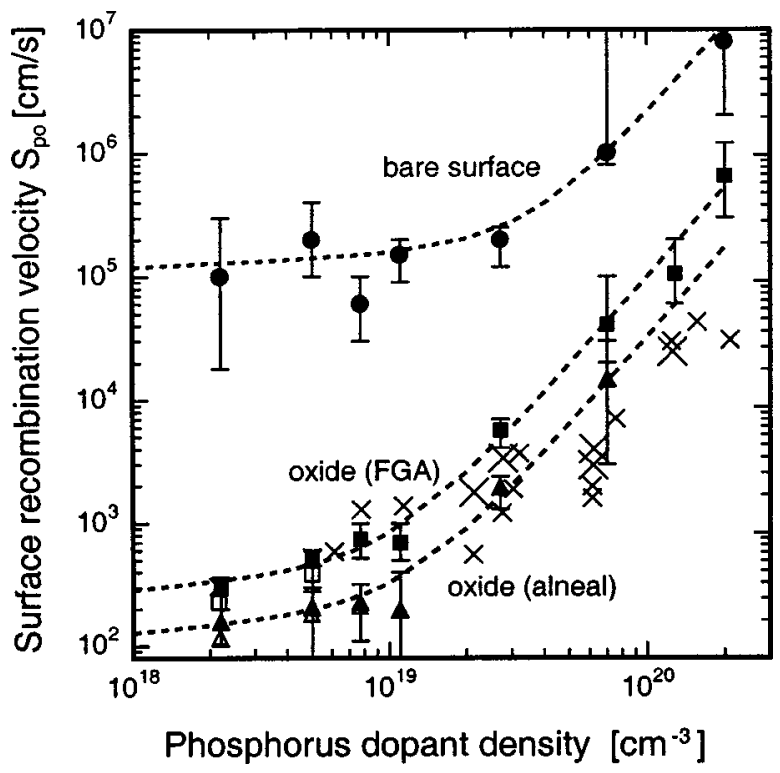

FIG. 7. Surface recombination velocity $S_{p o}$, as extracted by our simulations from the $J_{o e}$ measurements made by Cuevas et al. (Refs. 6-8) (symbols). The data obtained by their model, using Boltzmann statistics, are shown as crosses. The dashed lines are a parameterization with Eq. (7).

the planar emitters fabricated by Cuevas et al. ${ }^{6-8}$ Their emitters had either a bare surface, or were passivated by a thermal oxide with a forming gas anneal (FGA) or with an aluminum anneal (alneal). For details of the processing and measurement conditions, see Refs. 6-8.

Figure 7 shows that towards high $N_{\text {surf }}, S_{p o}$ increases in a straight manner, regardless of which type of surface passivation has been applied (all the dashed lines in Figs. 7-11 are a parameterization introduced in Sec. V C). Cuevas et al. used an analytical model together with apparent BGN and

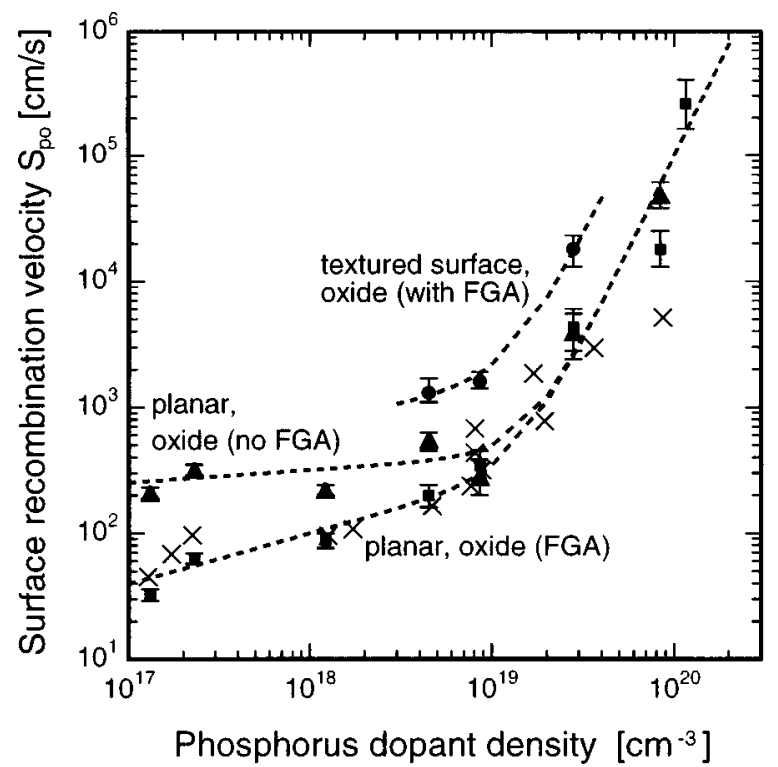

FIG. 8. Surface recombination velocity $S_{\text {po }}$, as extracted by our simulations from the $J_{o e}$ measurements made by King et al. (Ref. 12). As these authors used the old value of the intrinsic carrier density, Cuevas et al. (Refs. 6-8) revised their data with the new value, still using Boltzmann statistics (crosses). The dashed lines are a parameterization with Eq. (7). 


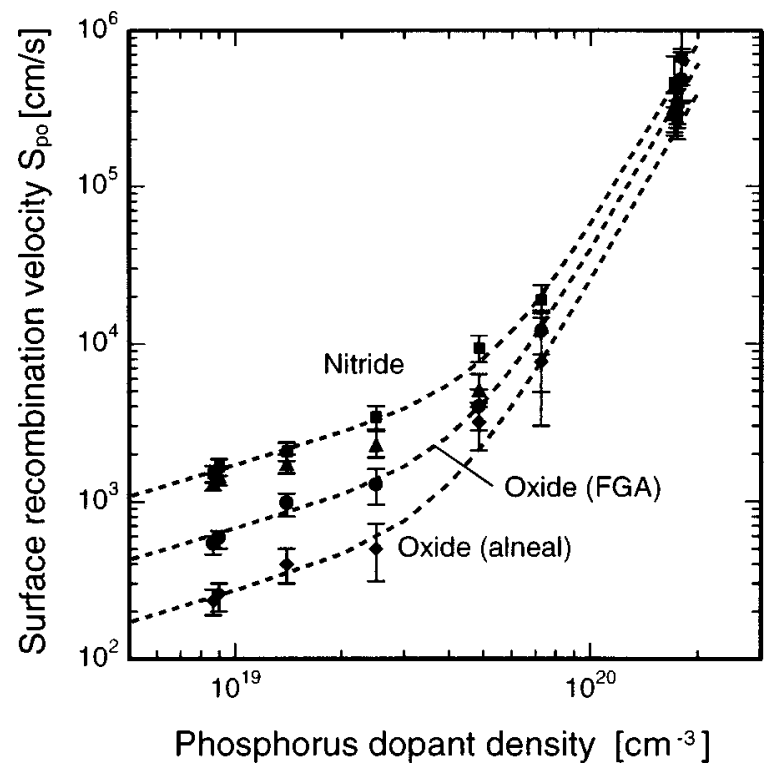

FIG. 9. Surface recombination velocity $S_{p o}$, as extracted by our simulations from the $J_{o e}$ measurements made by Kerr et al. The dashed lines are a parameterization with Eq. (7). For clarity, the parameterization of the oxide without FGA (triangles) is not shown.

Boltzmann statistics, and therefore obtained significantly lower $S_{p o}$ values at $N_{\text {surf }} \geq 3 \times 10^{19} \mathrm{~cm}^{-3}$ (indicated by the crosses in Fig. 7). As outlined in Sec. II, this was generally experienced in previous models, because degeneracy effects were not taken fully into account, leading to overestimated bulk recombination losses that were compensated with low $S_{p o}$ values (due to this compensation, previous models sometimes yielded negative $S_{p o}$ values in very highly doped emitters).

The same tendency is observed in our evaluation of the data from King et al., ${ }^{12}$ depicted in Fig. 8. For comparison, we have not plotted the original values of King et al. (be-

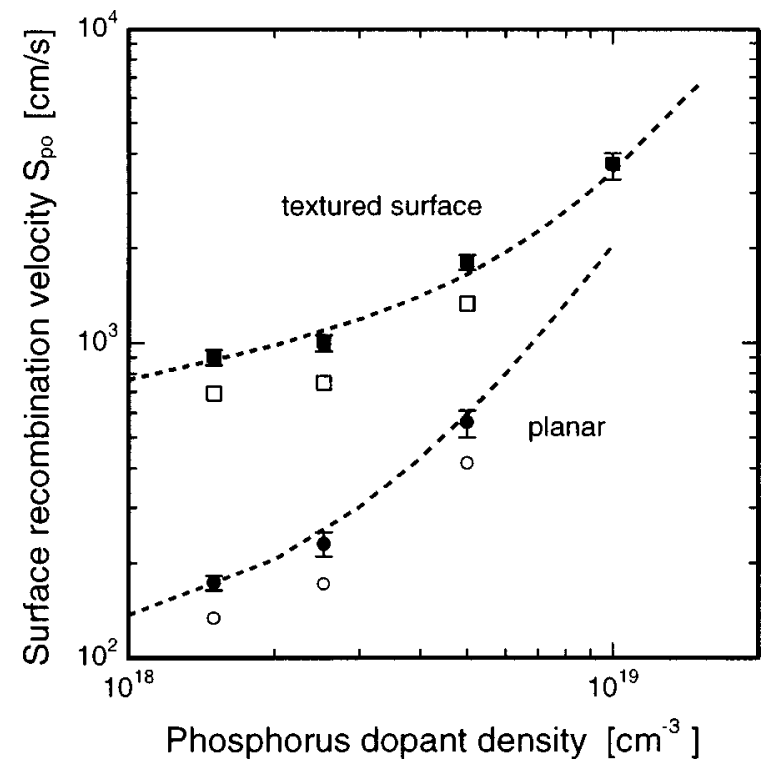

FIG. 10. Surface recombination velocity $S_{p o}$, as extracted by our simulations from the $J_{o e}$ measurements made by Glunz et al. (Ref. 9). The dashed lines are a parameterization with Eq. (7).

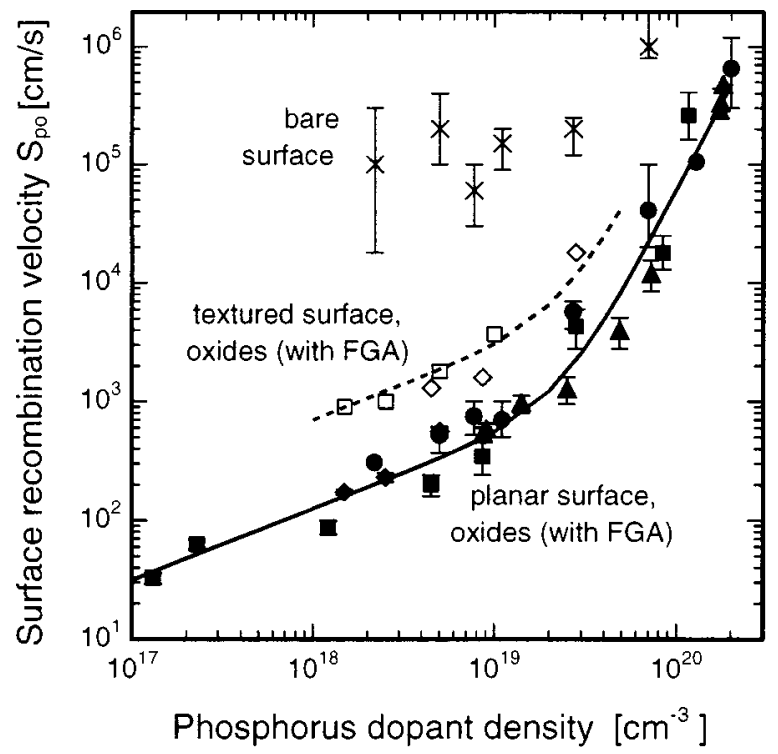

FIG. 11. Summary of Figs. 7-10, and parameterizations with Eq. (7).

cause they were obtained using the old $n_{i}$, see Sec. III A), but rather the revised values obtained with the analytical model of Cuevas et al. and the new $n_{i}$. Again, our $S_{p o}$ is significantly higher at high dopant densities due to degeneracy effects.

At the lowest $N_{\text {surf }}$, the injection conditions at the surface changed during the transient measurements performed by King $e t a l$., and we expect $J_{o e}$ to be injection dependent, as is commonly experienced in lightly doped rear surfaces of solar cells. ${ }^{90-92}$ We therefore obtained the $S_{p o}$ values only from the lowest injection levels in King's experiment (in contrast, more highly doped emitters remained in very low injection conditions during the measurement, so $J_{o e}$ is independent of the injection level).

Figure 9 shows $S_{p o}$ of the samples fabricated by Kerr et al. ${ }^{10,11}$ In the case of oxide passivated surfaces, very similar behavior is found in the samples of Cuevas et al. and King et al. Kerr et al. also investigated PECVD silicon nitride passivation and found that it results in a higher $S_{p o}$ than does oxide passivation at all investigated dopant densities, i.e., at $N_{\text {dop }}>9 \times 10^{18} \mathrm{~cm}^{-3}$. This remains in contrast to the common findings at low-doped surfaces, and suggests that the silicon nitride passivation is strongly based on electrostatic action, which works more efficiently at low dopant densities. Our analysis of the data for silicon nitride passivation includes a fixed positive oxide charge density of 2 $\times 10^{11} \mathrm{~cm}^{-2}$. In comparison we use $7 \times 10^{10} \mathrm{~cm}^{-2}$ at the $\mathrm{Si} / \mathrm{SiO}_{2}$ interface. The charge density has a negligible influence on our results, because the abundance of electrons in the emitters compensates for the fixed charges of the oxide or nitride layer very effectively.

So far, we have dealt with planar emitters. King et al. and Glunz et al. ${ }^{9}$ fabricated emitters in parallel on planar and pyramidically textured wafers, and our evaluation of their measurements are shown in Figs. 8 and 10. We consider only the emitters of Glunz et al. that were unaffected by nonhomogeneous dopant distributions, which may be found at textured surfaces in low diffusion profiles. In both data sets, we 
TABLE I. Parameters in Eq. (7) to approximate the $S_{p o}$ values shown in Figs. 7-10.

\begin{tabular}{|c|c|c|c|c|c|c|}
\hline & Cuevas et al. & & & King et al. & & \\
\hline & alneal & FGA & bare & FGA & bare & textured \\
\hline$S_{p 1}$ & 200 & 450 & $1.5 \times 10^{5}$ & 250 & 400 & 1500 \\
\hline$N_{p 1}$ & $1 \times 10^{19}$ & $1 \times 10^{19}$ & $1 \times 10^{19}$ & $1 \times 10^{19}$ & $1 \times 10^{19}$ & $1 \times 10^{19}$ \\
\hline$\gamma_{p 1}$ & 0.2 & 0.2 & 0.1 & 0.4 & 0.1 & 0.3 \\
\hline$S_{p 2}$ & 130 & 400 & 8000 & 100 & 100 & 700 \\
\hline$N_{p 2}$ & $1 \times 10^{19}$ & $1 \times 10^{19}$ & $1 \times 10^{19}$ & $1 \times 10^{19}$ & $1 \times 10^{19}$ & $1 \times 10^{19}$ \\
\hline \multirow[t]{3}{*}{$\gamma_{p 2}$} & 2.4 & 2.4 & 2.4 & 3 & 3 & 3 \\
\hline & \multicolumn{2}{|l|}{ Glunz et al. } & \multicolumn{4}{|l|}{ Kerr et al. } \\
\hline & textured & planar & nitride & bare oxide & FGA & alneal \\
\hline$S_{p 1}$ & 1500 & 250 & 1700 & 1400 & 670 & 270 \\
\hline$N_{p 1}$ & $1 \times 10^{19}$ & $1 \times 10^{19}$ & $1 \times 10^{19}$ & $1 \times 10^{19}$ & $1 \times 10^{19}$ & $1 \times 10^{19}$ \\
\hline$\gamma_{p 1}$ & 0.3 & 0.3 & 0.565 & 0.5 & 0.65 & 0.65 \\
\hline$S_{p 2}$ & 2000 & 1800 & 5 & 4 & 4 & 2.5 \\
\hline$N_{p 2}$ & $1 \times 10^{19}$ & $1 \times 10^{19}$ & $1 \times 10^{19}$ & $1 \times 10^{19}$ & $1 \times 10^{19}$ & $1 \times 10^{19}$ \\
\hline$\gamma_{p 2}$ & 2.2 & 2.2 & 4 & 4 & 4 & 4 \\
\hline
\end{tabular}

observe an approximately fivefold increase in $S_{p o}$ due to texturing. This increase is partly attributed to the 1.7 times larger surface area of textured compared to planar surfaces, which is not taken into account in our simulations. Partly, the increase may also be caused by the higher bond density of (111) surfaces in textured structures, compared to the (100) surface in planar surfaces.

Finally, we test by means of the lowest doped sample of Cuevas et al., how strongly the Coulomb enhancement of Auger recombination affects the extraction of $S_{p o}$. The omission of Coulomb enhancement increases the Auger losses in the bulk, and hence lowers $S_{p o}$, but only by about $3 \%$, and even less in more highly doped samples. These deviations are far smaller than the limitations imposed by the reproducibility of the experiments, and can be neglected.

Incomplete ionization has a slightly stronger effect in low-doped emitters, as is shown by the empty symbols in Figs. 7 and 10. However, as with the Coulomb enhancement, incomplete ionization generally has a lesser effect on $S_{p o}$ than the limitations imposed by the reproducibility of the experiments, and may be neglected as well.

\section{Interpretation of the results}

The $S_{p o}$ values, obtained from all the measurements, increase more gradually at low than at high dopant densities. This behavior is approximated by the following parameterization:

$$
S_{p o}=S_{p 1}\left(\frac{N_{\mathrm{dop}}}{N_{p 1}}\right)^{\gamma_{p 1}}+S_{p 2}\left(\frac{N_{\mathrm{dop}}}{N_{p 2}}\right)^{\gamma_{p 2}} .
$$

The values of the parameters $S_{p}$ (in units of $\mathrm{cm} / \mathrm{s}$ ), $N_{p}$ (in $\mathrm{cm}^{-3}$ ), and $\gamma_{p}$ (a number) are given in Table I for each data set. The parameter $\gamma_{p}$ is a measure for the slope, while both the values $S_{p}$ and $N_{p}$ shift $S_{p o}$ vertically, and hence are not uniquely defined; we choose $N_{p}=1 \times 10^{19} \mathrm{~cm}^{-3}$ for all the data sets. The data of Glunz et al. are restricted to the transition region between $N_{\text {dop }}$ where the first term in Eq. (7) dominates, and $N_{\text {dop }}$ where the second term dominates.
Therefore, they are most ambiguously fitted. Interestingly, the data from every group can be fitted with only one $\gamma_{p 2}$ for all the different passivation schemes. The values in Table I reflect that the $S_{p o}$ values vary among different authors to some extent. It is well known that the degree of surface passivation, and hence $S_{p o}$, depends on the processing conditions and also on technological factors, some of which are difficult to control. The original references describe that different oxidation conditions (temperatures in the range 900$1000{ }^{\circ} \mathrm{C}$ ) and $\mathrm{SiO}_{2}$ thicknesses (from 13 to $105 \mathrm{~nm}$ ) were used. In some cases, the oxide was grown in situ in the phosphorus diffusion furnace, while in other cases it was grown in a separate step. The presence or absence of TCA during the oxidation may also affect the results. Hence, the different data sets may indicate different degrees of surface passivation. However, these variations should not be overemphasized. Figure 11 summarizes the $S_{p o}$ values found with our improved analysis and demonstrates that the different data sets are in fact very similar. In fact, we may globally fit our planar oxidized samples that obtained a FGA with $S_{p 1}$ $=500$ and $S_{p 2}=60 \mathrm{~cm} / \mathrm{s}$, and our textured samples with $S_{p 1}=2800$ and $S_{p 2}=300 \mathrm{~cm} / \mathrm{s}$. For both surface structures, we use $\gamma_{p 1}=0.6, \gamma_{p 2}=3$, and $N_{p 1}=N_{p 2}=1 \times 10^{19} \mathrm{~cm}^{-3}$. Note that, due to the lack of data at high $N_{\text {surf }}, \gamma_{p 2}$ and $S_{p 2}$ are rather uncertain for textured samples. We emphasize that the surface parameters derived in this article should only be applied to device simulation if Fermi-Dirac statistics are used, together with the corresponding material parameters (such as $\Delta E_{g}$ instead of $\Delta E_{g}^{\mathrm{app}}$ ).

\section{CONCLUSIONS}

We have established a simulation model for phosphorusdoped silicon emitters using Fermi-Dirac statistics. Our model is based on a set of independently measured material parameters and on quantum mechanical calculations. In contrast with common simulations, which use Boltzmann statistics and apparent band-gap narrowing data, we use FermiDirac statistics and quantum mechanically derived band 
shifts, and therefore we account for the degeneracy effects on a physically sounder basis. This leads to unprecedented consistency even at very high dopant densities, and enables us to simulate emitters with $N_{\text {dop }}>3 \times 10^{19} \mathrm{~cm}^{-3}$ considerably more precisely than in the past.

With our improved model, we determined the hole surface recombination velocity $S_{p o}$ by reevaluating a broad range of measurements of the emitter saturation current density. The resulting $S_{p o}$ values increase with a power-law dependence on the dopant density of the surface $N_{\text {surf }}$, with the trend continuing at the highest dopant densities. This contrasts common models that use Boltzmann statistics and therefore needed to compensate for the neglected degeneracy effects by apparent band-gap narrowing and reduced $S_{p o}$ values. Despite small differences in oxide quality among the various laboratories, $S_{p o}$ generally increases more gradually at low than at high dopant densities. Also, pyramidal surface texturing increases $S_{p o}$ approximately by a factor of five. The parameterization of $S_{p o}$ given here completes the physical description of highly doped Si:P required for device modeling using advanced computer simulations.

\section{ACKNOWLEDGMENTS}

P.P.A. is on a Postdoctoral Fellowship from the Australian Research Council (ARC). The Center for Photovoltaic Engineering is supported by ARC's Special Research Centres Scheme. A.C. and M.K. also acknowledge funding by the ARC.

${ }^{1}$ H. Kroemer, RCA Rev. 28, 332 (1957).

${ }^{2}$ M. S. Lundstrom, R. J. Schwartz, and J. L. Gray, Solid-State Electron. 24, 195 (1981).

${ }^{3}$ F. A. Lindholm and J. G. Fossum, IEEE Electron Device Lett. 2, 230 (1981).

${ }^{4}$ A. H. Marshak, M. Ayman-Shibib, J. G. Fossum, and F. A. Lindholm, IEEE Trans. Electron Devices 28, 293 (1981).

${ }^{5}$ P. P. Altermatt, A. Schenk, and G. Heiser, J. Appl. Phys. (to be published).

${ }^{6}$ A. Cuevas, G. Giroult-Matlakowski, P. A. Basore, C. DuBois, and R. R. King, in Proceedings of the First World Conference on Photovoltaic Energy Conversion, Waikoloa, HI, pp. 1446-1449.

${ }^{7}$ A. Cuevas, P. A. Basore, G. Giroult-Matlakowski, and C. DuBois, in Proceedings of the 13th European Photovoltaic Solar Energy Conference, Nice, France (H.S. Stephens, Bedford, UK, 1995), pp. 337-340.

${ }^{8}$ A. Cuevas, P. A. Basore, G. Giroult-Matlakowski, and C. Dubois, J. Appl. Phys. 80, 3370 (1996).

${ }^{9}$ S. W. Glunz, S. Sterk, R. Steeman, W. Warta, J. Knobloch, and W. Wettling, in Proceedings of the 13th European Photovoltaic Solar Energy Conference, (Nice, France) (H.S. Stephens, Bedford, UK, 1995), pp. 409412.

${ }^{10}$ M. J. Kerr, J. Schmidt, and A. Cuevas, in Proceedings of the 16th EC Solar Energy Conference, Glasgow, UK (James \& James, London, 2000), pp. 1715-1718.

${ }^{11}$ M. J. Kerr, J. Schmidt, A. Cuevas, and J. H. Bultman, J. Appl. Phys. 89, 3821 (2001).

${ }^{12}$ R. R. King, R. A. Sinton, and R. M. Swanson, IEEE Trans. Electron Devices 37, 365 (1990).

${ }^{13}$ A. Schenk, J. Appl. Phys. 84, 3684 (1998).

${ }^{14}$ P. P. Altermatt, G. Heiser, A. G. Aberle, A. Wang, J. Zhao, S. J. Robinson, S. Bowden, and M. A. Green, Prog. Photovoltaics 4, 399 (1996).

${ }^{15}$ P. P. Altermatt, G. Heiser, X. Dai, J. Jürgens, A. G. Aberle, S. J. Robinson, T. Young, S. S. Wenham, and M. A. Green, J. Appl. Phys. 80, 3574 (1996).

${ }^{16}$ P. P. Altermatt, G. Heiser, K. McIntosh, T. Kiesewetter, C. B. Honsberg, S. R. Wenham, and M. A. Green, in Proceedings of the 26th IEEE Photovoltaic Specialists Conference (Anaheim, CA, USA), pp. 179-182, IEEE, Sept. 1997.
${ }^{17}$ P. P. Altermatt, J. Schmidt, G. Heiser, and A. G. Aberle, J. Appl. Phys. 82, 4938 (1997)

${ }^{18}$ P. P. Altermatt, A. Schenk, M. A. Green, and G. Heiser, in Technical Digest of the 11th International Photovoltaic Science and Engineering Conference, Sapporo, Japan (Tanaka, Kyoto, Japan, 1999), pp. 719-722.

${ }^{19}$ P. P. Altermatt, J. Schmidt, M. Kerr, G. Heiser, and A. G. Aberle, in Proceedings of the 16th European Photovoltaic Solar Energy Conference, Glasgow, UK (James \& James, London, 2000), pp. 243-246.

${ }^{20}$ P. P. Altermatt, R. A. Sinton, and G. Heiser, Sol. Energy Mater. Sol. Cells 65, 149 (2001).

${ }^{21}$ P. P. Altermatt, A. Schenk, F. Geelhaar, G. Heiser, and M. A. Green, J. Appl. Phys. (submitted).

${ }^{22}$ P. P. Altermatt, G. Heiser, and A. Schenk, J. Appl. Phys. (to be published).

${ }^{23}$ A. G. Aberle, P. P. Altermatt, G. Heiser, S. J. Robinson, A. Wang, J. Zhao, U. Krumbein, and M. A. Green, J. Appl. Phys. 77, 3491 (1995).

${ }^{24}$ J. O. Schumacher, P. P. Altermatt, G. Heiser, and A. G. Aberle, Sol. Energy Mater. Sol. Cells 65, 95 (2001).

${ }^{25}$ S. W. Glunz, J. Dicker, and P. P. Altermatt, Proceedings of the 17th EU Photovoltaic Energy Conference, Munich, Germany (WIP-Renewable Energies, Munich, Germany, 2001).

${ }^{26}$ P. P. Altermatt, J. O. Schumacher, A. Cuevas, S. W. Glunz, R. R. King, G. Heiser, and A. Schenk, in Proceedings of the 16th EC Solar Energy Conference, Glasgow, UK (James \& James, London, 2000), pp. 102-105.

${ }^{27}$ A. Schenk, Advanced Physical Models for Silicon Device Simulation (Springer, Wien, 1998).

${ }^{28}$ M. A. Green, J. Appl. Phys. 67, 2944 (1990).

${ }^{29}$ A. B. Sproul and M. A. Green, J. Appl. Phys. 70, 846 (1991).

${ }^{30}$ A. B. Sproul and M. A. Green, J. Appl. Phys. 73, 1214 (1993).

${ }^{31}$ K. Misiakos and D. Tsamakis, J. Appl. Phys. 74, 3293 (1993).

${ }^{32}$ D. B. M. Klaassen, J. W. Slotboom, and H. C. de Graaff, Solid-State Electron. 35, 125 (1992).

${ }^{33}$ R. R. King and R. M. Swanson, IEEE Trans. Electron Devices 38, 1399 (1991).

${ }^{34}$ J. W. Slotboom and H. C. de Graaff, Solid-State Electron. 19, 857 (1976).

${ }^{35}$ S. E. Swirhun, Y. H. Kwark, and R. M. Swanson, in International Electronic Devices Meeting, Los Angeles, CA (IEEE, New York, 1986), pp. 24-27.

${ }^{36}$ D. D. Tang, IEEE Trans. Electron Devices 27, 563 (1980).

${ }^{37}$ R. P. Mertens, J. L. van Meerbergen, J. F. Nijs, and R. J. van Overstraeten, IEEE Trans. Electron Devices 27, 949 (1980).

${ }^{38}$ A. Neugroschel, S. C. Pao, and F. A. Lindholm, IEEE Trans. Electron Devices 29, 894 (1982).

${ }^{39}$ D. S. Lee and J. G. Fossum, IEEE Trans. Electron Devices 30, 626 (1983).

${ }^{40}$ A. Wieder, IEEE Trans. Electron Devices 27, 1402 (1980).

${ }^{41}$ H. E. J. Wulms, IEEE J. Solid-State Circuits 12, 143 (1977)

${ }^{42}$ J. del Alamo and R. M. Swanson, Solid-State Electron. 30, 1127 (1987).

${ }^{43}$ G. E. Possin, M. S. Adler, and B. J. Baliga, IEEE Trans. Electron Devices 31, 3 (1984).

${ }^{44}$ S. E. Aw, H. S. Tan, and C. K. Ong, J. Phys.: Condens. Matter 3, 8213 (1991).

${ }^{45}$ M. Balkansi, A. Aziza, and E. Amzallag, Phys. Status Solidi 31, 323 (1969).

${ }^{46}$ P. E. Schmid, Phys. Rev. B 23, 5531 (1981).

${ }^{47}$ A. A. Volvson and V. K. Subashiev, Sov. Phys. Semicond. 1, 327 (1967).

${ }^{48}$ E. Daub and P. Würfel, J. Appl. Phys. 80, 5325 (1996).

${ }^{49}$ J. A. del Alamo, R. M. Swanson, and A. Lietoila, Solid-State Electron. 26, 483 (1983).

${ }^{50}$ H. P. D. Lanyon, A. K. McCurdy, and R. A. Tuft, in Proceedings of the 13th IEEE Photovoltaic Specialists Conference, Washington, DC (IEEE, New York, 1978), pp. 60-65.

${ }^{51}$ A. Neisser, Master's thesis, Institut für Festkörperphysik, Technical University of Berlin, 1998.

${ }^{52}$ J. Wagner and J. A. del Alamo, J. Appl. Phys. 63, 425 (1988).

${ }^{53}$ W. van Roosbroeck, Bell Syst. Tech. J. 29, 560 (1950).

${ }^{54}$ J. Beck and R. Conradt, Solid State Commun. 13, 93 (1973).

${ }^{55}$ J. Dziewior and W. Schmid, Appl. Phys. Lett. 31, 346 (1977).

${ }^{56}$ R. Häcker and A. Hangleiter, J. Appl. Phys. 75, 7570 (1994).

${ }^{57}$ R. M. Swanson and S. E. Swirhun, Tech. Rep., Sandia National Laboratories, 1987, Report No. SAND97-7019.

${ }^{58}$ A. Hangleiter and R. Häcker, Phys. Rev. Lett. 65, 215 (1990).

${ }^{59}$ M. J. Kerr and A. Cuevas, J. Appl. Phys. 91, 2473 (2002).

${ }^{60}$ W. Shockley and W. Read, Phys. Rev. 87, 835 (1952).

${ }^{61}$ R. Hall, Phys. Rev. 87, 387 (1952).

${ }^{62}$ A. Schenk, J. Appl. Phys. 79, 814 (1996). 
${ }^{63}$ J. del Alamo, S. Swirhun, and R. M. Swanson, in International Electron Devices Meeting, Washington DC (IEEE, New York, 1985), pp. 290-293.

${ }^{64}$ J. Dziewior and D. Silber, Appl. Phys. Lett. 35, 170 (1979).

${ }^{65}$ B. Krüger, T. Friese, F. El-Ratel, W. Sharabati, and H. G. Wagemann, Solid-State Electron. 39, 897 (1996).

${ }^{66}$ G. W. Ludwig and R. L. Watters, Phys. Rev. 101, 1699 (1956).

${ }^{67}$ J. Müller, H. Bernt, and H. Reichl, Solid-State Electron. 21, 999 (1978).

${ }^{68}$ A. Neugroschel, IEEE Electron Device Lett. 6, 425 (1985).

${ }^{69}$ M. B. Prince, Phys. Rev. 93, 1204 (1954).

${ }^{70}$ A. B. Sproul, M. A. Green, and A. W. Stephens, J. Appl. Phys. 72, 4161 (1992).

${ }^{71}$ E. Susi, L. Passari, M. Merli, and M. C. Carotta, Phys. Status Solidi A 106, 583 (1988).

${ }^{72}$ S. E. Swirhun, J. A. del Alamo, and R. M. Swanson, IEEE Electron Device Lett. 7, 168 (1986).

${ }^{73}$ C. H. Wang and A. Neugroschel, IEEE Trans. Electron Devices 11, 576 (1990).

${ }^{74}$ C. H. Wang, K. Misiakos, and A. Neugroschel, Appl. Phys. Lett. 57, 159 (1990).

${ }^{75}$ C. H. Wang, K. Misiakos, and A. Neugroschel, IEEE Trans. Electron Devices 37, 1314 (1990).

${ }^{76}$ M. Zerbst and W. Heywang, Z. Naturf. A 11, 608 (1956).

${ }^{77}$ D. B. M. Klaassen, Solid-State Electron. 35, 953 (1992).

${ }^{78}$ J. A. del Alamo and R. M. Swanson, IEEE Trans. Electron Devices 31, 1878 (1984).

${ }^{79}$ M. H. Cohen, M. Y. Chou, E. N. Ecoonomou, S. Jahn, and C. M. Soukoulis, IBM J. Res. Dev. 32, 82 (1988).
${ }^{80}$ J. Serre and A. Ghazali, Phys. Rev. B 28, 4704 (1983).

${ }^{81}$ A. Ghazali and J. Serre, Solid-State Electron. 28, 145 (1985).

${ }^{82}$ Y. Pan, S. C. Jain, M. Kleffstra, and P. Balk, Solid-State Electron. 35, 791 (1992).

${ }^{83}$ Y. Pan, J. Appl. Phys. 70, 838 (1991).

${ }^{84}$ C. H. Grein and S. John, Phys. Rev. B 36, 7457 (1987).

${ }^{85}$ M. Levy, P. Y. Yu, Y. Zhang, and M. P. Sarachik, Phys. Rev. B 49, 1677 (1994).

${ }^{86} \mathrm{~S}$. Integrated Systems Engineering, AG, Zurich, Dessis manual version 6.1, 2000.

${ }^{87}$ G. Masetti, D. Nobili, and S. Solmi, in Semiconductor Silicon 1977, Proceedings of the Third International Symposium on Silicon Materials Science and Technology edited by H. R. Huff and E. Sirtl (The Electrochemical Society, 1977), pp. 648-657.

${ }^{88}$ G. Ottaviani, L. Reggiani, C. Canali, F. Nava, and A. Alberigi-Quaranta, Phys. Rev. B 12, 3318 (1975).

${ }^{89}$ C. Canali, C. Jacoboni, F. Nava, G. Ottaviani, and A. Alberigi-Quaranta, Phys. Rev. B 12, 2265 (1975).

${ }^{90}$ S. J. Robinson, S. R. Wenham, P. P. Altermatt, A. G. Aberle, G. Heiser, and M. A. Green, J. Appl. Phys. 78, 4740 (1995).

${ }^{91}$ A. G. Aberle, S. Glunz, and W. Warta, J. Appl. Phys. 71, 4422 (1992).

${ }^{92}$ S. W. Glunz, A. B. Sproul, W. Warta, and W. Wettling, J. Appl. Phys. 75, 1611 (1994).

${ }^{93}$ G. Masetti, M. Severi, and S. Solmi, IEEE Trans. Electron Devices 30, 764 (1983). 